\title{
EFFECTS OF CONCURRENT CONTINUOUS VISUAL FEEDBACK ON LEARNING THE LANE KEEPING TASK
}

\author{
Peter van Leeuwen, Stefan de Groot, Riender Happee, \& Joost de Winter \\ Biomechanical Engineering, Faculty of Mechanical, Maritime and Materials Engineering, \\ Delft University of Technology \\ Delft, The Netherlands \\ Email: P.M.vanLeeuwen@TUDelft.nl
}

\begin{abstract}
Summary: This study investigated the training effectiveness of continuous visual feedback in a simulator-based lane keeping task. Two groups of student drivers (total of 30 participants) were instructed to drive as accurately as possible in the center of the right lane in a self-paced driving task during five 8-min sessions. One group received visual feedback using a horizontal compensatory display positioned on the dashboard, which provided an indication of the momentary distance to the lane center during the three training sessions. During two retention sessions (immediate and one day delayed) both groups drove without the augmented feedback. The augmented feedback resulted in improved performance on a measure lane keeping accuracy, but this effect disappeared during retention. Furthermore, the augmented feedback resulted in increased steering wheel activity during all sessions, and increased driver workload in the delayed retention session. These results provide support for the guidance hypothesis and have possible implications for the use of continuous concurrent feedback in simulatorbased driver training.
\end{abstract}

\section{INTRODUCTION}

Nowadays, driving simulators are increasingly used in driver training programs (Allen, Park, Cook, \& Fiorentino, 2009; De Winter, De Groot, Mulder, Wieringa, Dankelman, \& Mulder, 2009). Driving simulators offer important advantages compared to on-the-road training, such as control and repeatability of training conditions, objective performance assessment, and guaranteed safety in difficult driving situations. Furthermore, with simulators it is possible to enhance the task intrinsic information by means of visual, auditory, or tactile augmented feedback.

Augmented feedback is information provided in addition to the task-intrinsic feedback and can be used to guide the learner to higher task performance during the training phase of skill acquisition. Augmented feedback can help to speed up the learning process, but is also known to lead to potential degradation of task performance when the augmented feedback is withdrawn in post training retention tests (Schmidt \& Wulf, 1997). This phenomenon is described by the guidance hypothesis (Salmoni, Schmidt, \& Walter, 1984). Too much guidance can result in learners not developing the information processing activity required to perform the task without augmented feedback, and to a development of over-corrective behavior (Young, Schmidt, \& Lee, 2001). Such maladaptive corrections occur if the augmented feedback evokes corrective actions that are trivially small or beyond the precision of the motor system (Lee \& Carnahan, 1990). Swinnen (1996) stated that augmented feedback can benefit learning when it is presented in such a way that learners do not become dependent on it. For simple motor tasks, continuous 
concurrent augmented feedback is known to be ineffective (Schmidt \& Wulf, 1997). However, for more complicated tasks, studies have shown that continuous concurrent feedback can improve performance during retention (Wulf \& Shea, 2002). A recent study concerning driver training using simulators has shown that training with augmented vibratory feedback results in faster learning and higher retention performance of the lane keeping task than training without augmented feedback (De Groot, De Winter, López-García, Mulder, \& Wieringa, 2011). In that study, the augmented feedback was designed such that the learner could not become dependent on it, using a bandwidth scheme, and by using binary (on/off) non-directional feedback.

The aim of this study was to investigate the effect of continuous concurrent visual feedback in simulator-based driver training. Lane keeping accuracy has been used as a measure to describe road safety in several studies (Brookhuis \& De Waard, 1993; Östlund et al., 2004) and is also the primary performance measure in this study. Continuous visual feedback on the momentary lane center deviation was presented on the dashboard of the virtual car. Learning performance was assessed during an immediate and a delayed retention session. Taking into account the effect of sleep on learning behavior (Walker, Brakefield, Morgan, Hobson, \& Stickgold, 2002) the second retention session took place one day after the training and immediate retention sessions.

\section{METHOD}

\section{Participants}

Thirty participants (21 men, 9 women) were tested in two groups. One group received augmented feedback (FB, 14 participants) during training and a control group drove without augmented feedback (NFB, 16 participants). Participants had no driver's license (13 participants were already taking driving lessons) and all participants were recruited from the student community. The mean age was 19.5 years $(S D=2.2)$ and all participants completed an intake questionnaire prior to participation in the experiment with the following variables: 1) Gender (male/female); 2) Possesion of a motorcycle or moped drivers licence (yes/no); 3) Experience in driving simulators (yes/no) and 4) Playing (minimum 1 hour per week) of video games (yes/no). Using the results of these variables the participants were assigned to one of the two groups using the minimization method of Taves (1974).

\section{Apparatus}

The simulator used for this study was the Green Dino driving simulator (Green Dino, 2010) which is used for initial driver training in The Netherlands. This fixed-base simulator, with 180degree field of view and surround sound simulates a middle class passenger vehicle. The simulator was equipped with realistic controls; the seat, pedals, and steering wheel originated from a real car. Steering force feedback was passive and the engine model represented that of a realistic car with automatic transmission.

The virtual world was projected using three LCD projectors with $1024 \times 768$ pixels for the center display and $800 \times 600$ pixels for the two side displays. The feedback (a horizontal compensatory display) was projected on the simulated dashboard. Instruments and mirrors were integrated in the simulation visualisation. 
To measure the amount of fixations and time drivers spent looking at the feedback area, a remote mounted Facelab eye tracker was used with two cameras mounted above the steering wheel and below the virtual scenery. See Figure 1 for an overview of the simulator, the eye tracker, and the feedback.

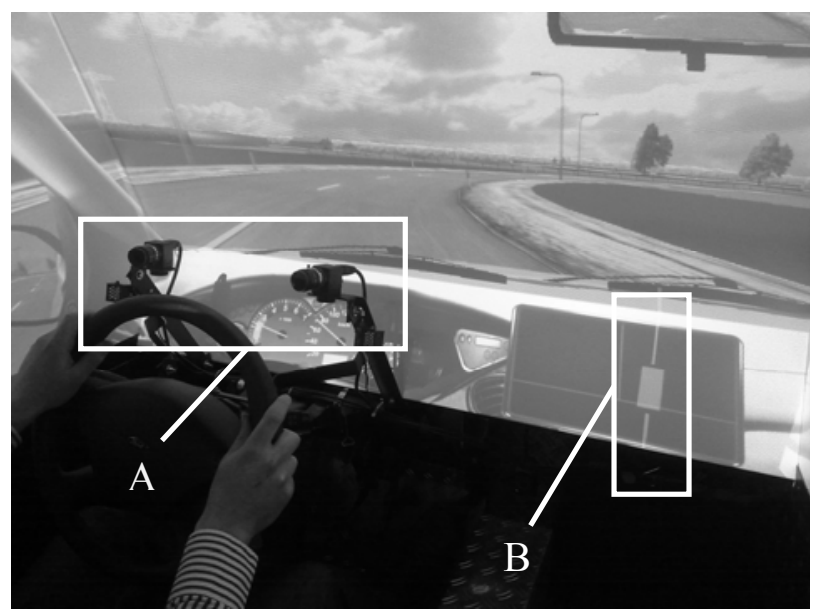

Figure 1. Simulator during the experiment

$(\mathrm{A}=$ eye tracker, $\mathrm{B}=$ augmented feedback; the vertical lines of the augmented feedback represent the lane center, while the rectangle indicates the vehicle position with respect to the lane center)

\section{Procedure and task}

Before participating in the experiment, participants completed an intake questionnaire for group assignment and were informed of the driving task. All participants provided written informed consent and the research was approved by the Human Research Ethics Committee of the Delft University of Technology. All sessions took place on a two-lane rural road of $7.5 \mathrm{~km}$ length, with varying curvature and without other traffic. The instructed task was to keep the vehicle as accurately as possible in the center of the right lane, while maintaining a realistic speed within the $80 \mathrm{kph}$ speed limit. Gear selection was automated; participants were required to steer, accelerate, and brake. Participants were informed of the presence of the eye tracker and, if applicable, the presence of the augmented feedback, explaining the visualization of the lane center error. Participants were not informed of the absence of the augmented feedback during the retention sessions. After being seated in the simulator the eye tracker was calibrated to each participant and the instructions were repeated on the screen.

Each participant drove three training sessions of eight minutes followed by a maximum three minute break, in which they completed the NASA TLX questionnaire for measuring workload (Hart \& Staveland, 1988). After the three training sessions an immediate retention session took place and a second retention session was driven one day later. During the retention sessions the augmented feedback was disabled. The eye tracker was recalibrated before the start of the last retention session. 


\section{Dependent measures}

To determine the effect of the augmented feedback on the lane keeping task the following dependent measures were determined for each training and retention session.

1. Root mean square error (RMSE) with respect to the lane center (m), describing the lane keeping accuracy.

2. Lane center error band; the time-percentage of total driving time that drivers kept the absolute lane center error smaller than 0.10 meters. This measure represents of the amount of near-perfect lane keeping accuracy.

3. Average speed $(\mathrm{m} / \mathrm{s})$ was included in this study as a measure of the participants' efficiency of completing the driving task. The speed over the complete course (including corners) was used in this study.

4. Steering wheel steadiness (\%); this measure was calculated as the percentage of the time the steering wheel's angular velocity was smaller than one degree per second. Reduced steering wheel steadiness is related to the increased amount of steering corrections.

5. Number of fixations on the feedback area, describing the number of times participants fixated on the feedback area. A single fixation was determined as a consecutive sequence of individual gaze points in the feedback area of $150 \mathrm{~ms}$ or longer (Salvucci \& Goldberg, 2000; Hornof \& Halverson, 2002).

6. NASA TLX, a subjective workload assessment tool in the form of a questionnaire.

The results were statistically compared per session between the FB and NFB groups using an independent two-sample $t$ test with $\alpha=0.05$. Missing eye tracker data (e.g., temporary loss of gaze tracking due to rapid head movements) were discarded from the analysis.

\section{RESULTS}

In Table 1, the mean values for the dependent measures are presented and the statistical significance between the NFB and FB groups per session is indicated with the $p$-values.

No significant difference was found in the RMSE lane center and average speed between the NFB and FB groups in both the training and retention sessions. The time participants kept the vehicle at a small lateral error was higher for the FB group during training session (significant in the third session), but this was not transferred to the retention session. Steering wheel steadiness was significantly higher for NFB in the training sessions, and this result was transferred to the retention sessions. The lane center error band and the steering wheel steadiness are illustrated in Figure 2. Steering wheel steadiness increased from the first training session to the second and remained relatively constant afterwards.

The number of fixations indicates that the FB group looked at the augmented feedback during training sessions. In the retention sessions, the FB group did not look significantly more to the augmented feedback area compared to the NFB group. A higher workload was reported by the FB group and this was significant $(p=.049)$ for the temporal demand item in the second retention session. The temporal demand item represents the time pressure felt due to the rate or pace at which the task or task elements occurred (Hart \& Staveland, 1988). 
Table 1. Averaged group results and corresponding $p$-values for NFB $(n=16)$ and FB $(n=14)$ groups in training and retention sessions

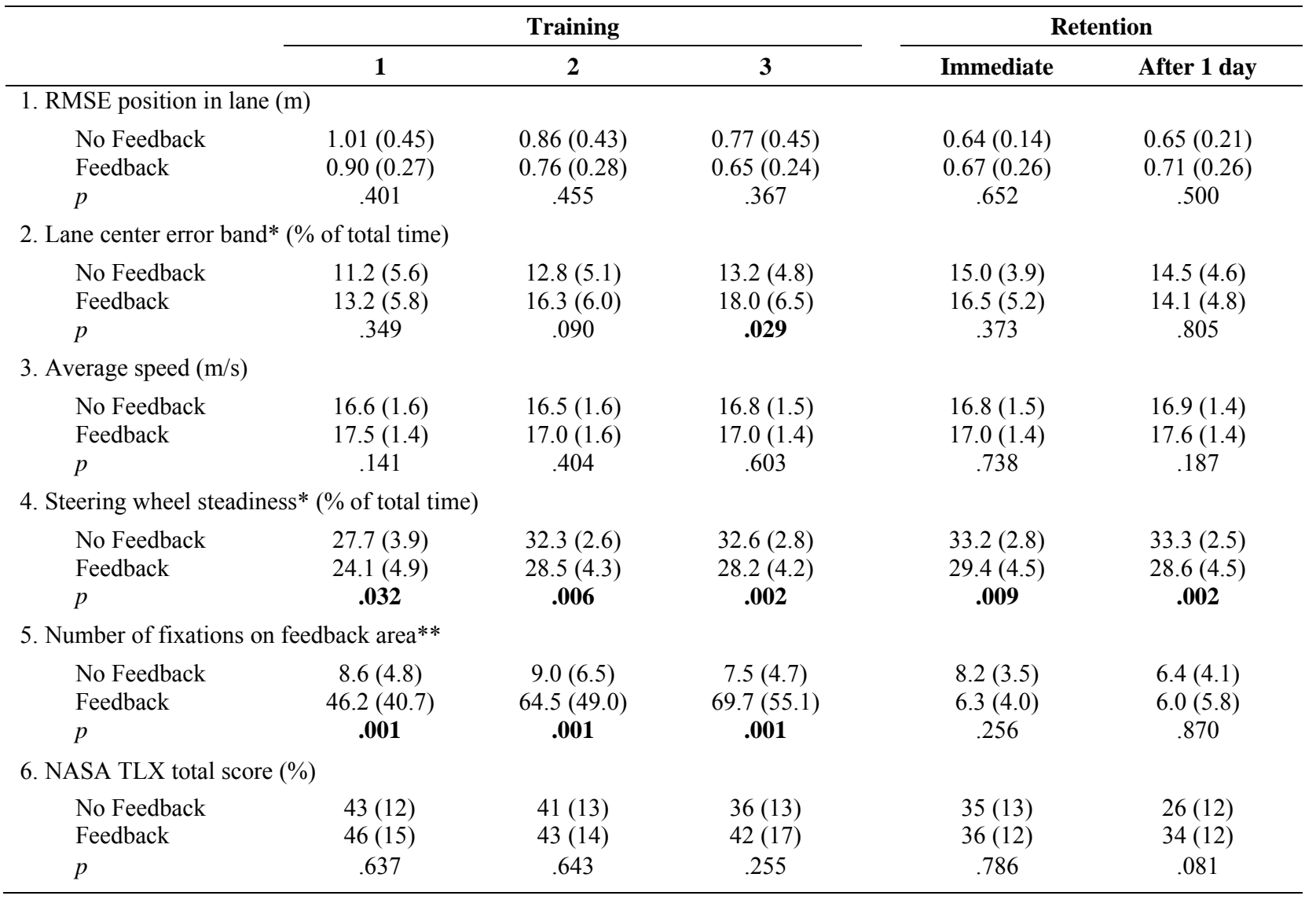

Note: standard deviations in parentheses

* Several other measures with various thresholds have been evaluated, providing similar results

** Not all data was valid, $64.5 \%$ of the data were included in the analysis
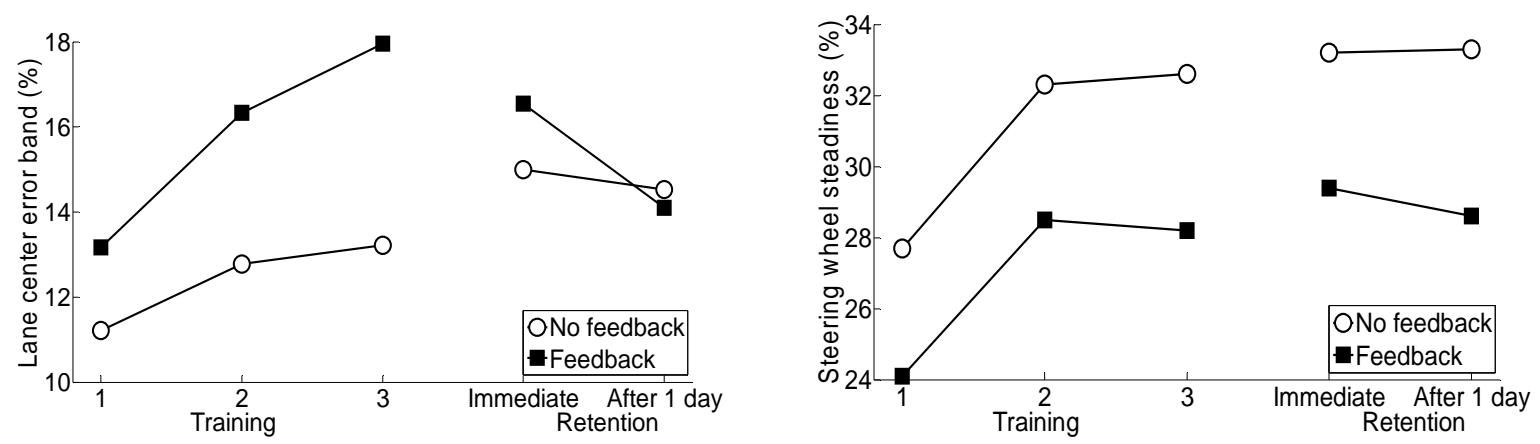

Figure 2. Lane center error band (left) and steering wheel steadiness (right) for both groups during training and retention sessions

\section{DISCUSSION}

In this study, we investigated the training effectiveness of continuous concurrent visual feedback in a simulator-based lane keeping task. Consistent with the guidance hypothesis, the total time participants drove very near to the lane center was higher for the FB group in training and these 
effects disappeared in the retention phase. The RMSE lateral error showed similar trends in both training and retention but these were not significant.

The FB group had a significantly reduced steering wheel steadiness during training as compared to the NFB group. This over-corrective steering behavior is related to the maladaptive correction hypothesis of augmented feedback (Lee \& Carnahan, 1990; Young et al., 2001). The FB group used more steering actions to keep a near-perfect lane keeping accuracy during training, and this effect persisted in retention when the augmented feedback was absent for both groups.

The concurrent augmented feedback might have overemphasized the trivially small momentary lane center errors. In contrast to the augmented feedback, the task-intrinsic visual feedback represents a combination of current and future lane center error as well as heading information (Donges, 1978; Land \& Horwood, 1995). The reduced perception of future error for the FB group may have led to reduced "anticipatory" driving behavior, resulting in a lower steering wheel steadiness and increased temporal demands.

The mean fixation time for the FB group was 0.64 seconds, indicating that participants used the augmented feedback as a short verification of the perceived lane center error; participants discretely sampled the augmented feedback as opposed to looking at the augmented feedback for longer subsequent periods.

In this study, the augmented feedback did not result in improved retention of lane keeping accuracy and the augmented feedback negatively influenced the steering control behavior and workload (temporal demands) of the participants. Because of these results, we recommend to be cautious with applying continuous concurrent visual feedback in driver training. For future work, different types of visual feedback could be considered, which reduce the dependency of the participant like bandwidth feedback (Lee \& Carnahan, 1990; De Groot et al., 2011), or which include the predicted future lane center error.

\section{ACKNOWLEDGMENTS}

We would like to thank David van den Berg, Bob van der Heijden, Ronald Immerzeel and Kasper Uithof for their efforts in setting up and conducting the experiments. The research is supported by the Dutch Ministry of Economic affairs through the project Driver Observation in Car Simulators (HTASI09004-DrivObs) and by the Stichting voor de Technische Wetenschappen (Dutch Technology Foundation) STW, applied science division of the Nederlandse Organisatie voor Wetenschappelijk Onderzoek (Netherlands Organisation for Scientific Research) NWO and the Technology Program of the Ministry of Economic Affairs.

\section{REFERENCES}

Allen, R.W., Park, G., Cook, M., \& Fiorentino, D. (2009). Training and assessment of novice drivers. Proceedings of the Driving Simulation Conference (DSC) 2009 Europe, 91-102.

Brookhuis, K.A., \& De Waard, D. (1993). The use of psychophysiology to assess driver status. Ergonomics, 36, 1099-1110. 
De Groot, S., De Winter, J.C.F., López-García, J.M., Mulder, M., \& Wieringa, P.A. (2011). The effect of concurrent bandwidth feedback on learning the lane keeping task in a driving simulator. Human Factors, 53, 50-62.

De Winter, J.C.F., De Groot, S., Mulder, M., Wieringa, P.A., Dankelman, J., \& Mulder, J.A. (2009). Relationships between driving simulator performance and driving test results. Ergonomics, 52, 137-153.

Donges, E. (1978). A two-level model of driver steering behavior. Human Factors, 20, 691-707.

Green Dino. (2010). Retrieved December 23, 2010, from http://www.dutchsimulators.com.

Hart, S.G., \& Staveland, L.E. (1988). Development of NASA-TLX (Task Load Index): Results of empirical and theoretical research. In: P. A. Hancock \& N. Meshkati (Eds.), Human Mental Workload (pp. 139-183). North-Holland: Elsevier Science.

Hornof, A.J., \& Halverson, T. (2002). Cleaning up systematic error in eye-tracking data by using required fixation locations. Behavior Research Methods, Instruments, and Computers, 34, 592-604.

Land, M.F., \& Horwood, J. (1995). Which parts of the road guide steering? Nature, 377, 339340.

Lee, T.D., \& Carnahan, H. (1990). Bandwidth knowledge of results and motor learning: More than just a relative frequency effect. The Quarterly Journal of Experimental Psychology, 42A, 777-789.

Östlund, J., Nilsson, L., Carsten, O., Merat, N., Jamson, H., Jamson, S. et al., (2004). Deliverable 2 - HMI and safety-related driver performance: Human Machine Interface And the Safety of Traffic in Europe (HASTE). Project Report No. GRD1/2000/25361 S12.319626.

Salmoni, A.W., Schmidt, R.A., \& Walter, C.B. (1984). Knowledge of results and motor learning: A review and critical reappraisal. Psychological Bulletin, 95, 355-386.

Salvucci, D.D., \& Goldberg J.H. (2000). Identifying fixations and saccades in eye-tracking protocols. Proceedings of the 2000 Symposium on eye tracking research and applications, 71-78.

Schmidt, R.A., \& Wulf, G. (1997). Continuous concurrent feedback degrades skill learning: Implications for training and simulation. Human Factors, 39, 509-525.

Swinnen, S.P. (1996). Information feedback for motor skill learning: A review. In H.N. Zelaznik (Ed.), Advances in motor learning and control (pp. 37-66). Champaign: Human Kinetics.

Taves, D.R. (1974). Minimization: A new method of assigning patients to treatment and control groups. Clinical Pharmacology and Therapeutics, 15, 443-453.

Walker, M.P., Brakefield, T., Morgan, A, Hobson, J.A., \& Stickgold, R. (2002). Practice with sleep makes perfect: Sleep-dependent motor skill learning. Neuron, 35, 205-211.

Wulf, G., \& Shea, C.H. (2002). Principles derived from the study of simple skills do not generalize to complex skill learning. Psychonomic Bulletin \& Review, 9, 185-211.

Young, D.E., Schmidt, R.A., \& Lee, T.D. (2001). Skill learning: Augmented feedback. In W. Karwowski (Ed.), International encyclopedia of ergonomics and human factors, 558-561. 